\title{
A-DIVA Score and the Implementation of the Use of Ultrasound-Guided Peripheral Intravenous Access in the Emergency Department: A Brief Literature Review
}

\author{
Annette D. Nacci ${ }^{1}$ \\ ${ }^{1}$ Emergency Department, Elkhart General Hospital, Elkhart, Indiana, USA \\ Correspondence: Samuel P. Abraham, Associate Professor of Nursing, 1001 Bethel Circle, Bethel College School \\ of Nursing, Mishawaka, Indiana, 46545, USA.
}

Received: November 15, 2018

Accepted: November 30, 2018

Online Published: December 4, 2018

doi:10.20849/ijsn.v3i3.517

URL: https://doi.org/10.20849/ijsn.v3i3.517

\begin{abstract}
Background: Peripheral intravenous (PIV) access is performed daily in the emergency department (ED). Difficult to access patients receive multiple intravenous attempts prior to successful cannulation. USGPIVA is a noninvasive technique to improve successful cannulation with the first attempt on a difficult to access patient. Use of the A-DIVA scoring method may determine difficult access patients prior to cannulation attempts. Purpose: The purpose of this literature review was to examine A-DIVA score and the implementation of the use of ultrasound-guided peripheral intravenous access in the emergency department. Method: A systematic review of USGIV was completed of the following databases: OVID, Current Index to Nursing and Allied Health Literature (CINAHL), PubMed, EBSCO host, and Google Scholar. Conclusion: A gap existed in the use of USGPIV in the ED. Therefore, the application of an A-DIVA score to implement USGPIV access may be beneficial. The A-DIVA prediction tool may improve the success rate of peripheral vascular access in adults rated to have difficult vascular access. Research studies on this important topic are recommended.
\end{abstract}

Keywords: difficult intravenous access, emergency department, ultrasound-guided peripheral intravenous, A-DIVA score, nursing action process, and knowledge translation

\section{Introduction}

Intravenous cannula placement is a procedure performed by nurses daily in the ED in multiple healthcare facilities and locations. A peripheral intravenous (PIV) catheter is placed to obtain blood specimens, administer fluids and medications. Not all patients needing PIV placement can be accessed on the first attempt. It is difficult to determine which patients will need one-attempt vs. multiple attempts for PIV. This may cause a delay in patient care and potentially a deterioration of a patient's condition (Adhikari, Schmier, \& Marx, 2015). Hospital vascular access teams (VAT) are not always an option for difficult to access patients. Therefore, techniques and technology to improve PIV success rates are warranted. As nurses become highly skilled using the USGPIV, wait times for results of diagnostic tests from lab and CAT scans should decrease. Improving patient satisfaction with PIV procedures may decrease the patient's stress, anxiety, and pain during placement. "When nurses can provide an improved experience with IV placement under ultrasound guidance, there are many associated positive effects on overall ED experience both from the perspective of the patient and the nurse" (Pandurangadu, Tucker, Bagan, $\&$ Bahl, 2016, p. 3). The purpose of this literature review was to examine A-DIVA score and the implementation of the use of ultrasound-guided peripheral intravenous access in the emergency department.

\section{Background and Problem}

At a northern Indiana rural hospital, the current practice for PIV access sometimes result in some patients receiving multiple attempts for a PIV. The current standard for PIV access is two attempts per ED nurse. There is no limit set on how many nurses attempt to obtain access. A patient's current medical condition and skill of the nurse may result in some patients receiving multiple PIV attempts from ED staff prior to contacting the VAT nurse. Approximately $30 \%$ of all PIV access performed in the ED will take 15 minutes, and difficult and challenging access may take as long as two hours (Witting, 2012). While this seems like a small percentage, the time consumed with multiple ED attempts and the wait for USGPIV from a VAT nurse will markedly delay a patient's treatment and care. 


\section{Literature Review}

A systematic review of USGIV was completed of the following databases: OVID, Current Index to Nursing and Allied Health (CINAHL), PubMed, EBSCOhost, and Google Scholar. The keywords used include difficult intravenous access, emergency department, ultrasound-guided peripheral intravenous, A-DIVA score, nursing action process, and knowledge translation. Few articles were noted in the recent nursing literature. The Journal of Vascular Access provided the most information regarding the subject of USGPIV. Also, articles published in the Journal of Emergency Nursing regarding ED utilization of USGPIV were limited. The nursing literature regarding A-DIVA scores resulted in two articles. It is noted that the pediatric literature did identify four articles on the development of DIVA scoring.

\subsection{Empirical Review}

PIV cannulation is a necessary procedure in an ED. Many patients are exposed to multiple PIV attempts. Risk factors have been identified to indicate which patients may be considered a difficult PIV access. Placement of PIV catheters at high flexion joints, increase the risk of phlebitis, thrombosis, and catheter infections (Sou et al., 2017).

In years past, a central venous catheter (CVC) was the standard in vascular access for a critically ill or injured patient in the ED. Physicians usually inserted these access devices with nursing assistance. The risk to the patient is greater with a CVC. Complications from the insertion of a CVC that were identified include infections, large artery puncture, and pneumothorax (Pandurangadu et al., 2016). This procedure is time-consuming for the staff and increases health care costs (Maiocco \& Coole, 2012). Moore (2013) noted, the implementation of the National Safety Goal 07.04.01 and the Joint Commission accreditation requirements, concluded the risks associated with placing a CVC outweigh the benefits. These provisions have created an increase in the number of PIV necessary in the ED.

There have been tools designed to help identify this patient population. The utilization of USGPIV in the ED setting has been demonstrated to reduce infection, patient perceived pain and improved patient satisfaction. Using the A-DIVA scale, patients can be identified as a difficult PIV access prior to multiple attempts. Van Loon, Puijn, Houterman, and Bouwman (2016), developed a predictive scale for difficult PIV access. The use of this predictive tool A-DIVA may increase the time-frame needed to use other techniques. The five identified risk factors are palpability of the target vein, visibility of the target vein, history of previous difficult PIV access, unplanned surgery, and a vein diameter of at most $2 \mathrm{~mm}$. A higher score on the A-DIVA indicates a higher risk for PIV placement.

Petroski, Frisch, Joseph, and Carlson (2015) stated that obtaining PIV access in an acceptable time with minimal attempts might improve both patient outcomes and satisfaction. Petroski et al. also noted that multiple IV attempts had been associated with increased pain. The study was initiated to identify predictors of difficult PIV access in the pediatric patient. They also attempted to identify if a difficult PIV will lead to delay in treatment.

This review may enhance the profession of nursing through an action process with implementation-based research for knowledge translation. The practice of multiple PIV attempts can be replaced with the use of the A-DIVA scale. This may promote the use of USGPIV with difficult PIV access patients as a first-line approach to PIV access. The knowledge of an ED nurse and skill-set may also be enhanced.

Nurses in the ED place PIV's multiple times during a shift. This is a fundamental nursing skill that can be difficult to perform. Carr et al. (2016a) identified 12 risk factors to predict insertion failure of a PIV in the ED. "These factors include: age, gender, race, body mass index, history of chemotherapy, diabetes, dialysis, intravenous drug abusers, swelling, non-visible veins, sickle cell disease and recent hospitalization or ED visit within 90 days" (p. 2). Patients with difficult PIV access are subjected to repeated attempts by multiple nurses. Pandurangadu et al. (2016) noted that "3.6 attempts for successful IV placement by blind techniques can require upwards of 120 minutes to obtain access when multiple providers are involved” (p. 3).

Maiocco and Coole (2012), in a 10-month study, demonstrated 51\% of obese patients needed PIV and $41 \%$ of them needed PIV restarts with 24 hours. These patients had multiple sticks greater than two attempts and needed peripheral intravenous central catheter (PICC) insertion. They also noted that there was a lack of trained staff to perform PICC insertions causing insertions to be delayed. To facilitate improvement in PIV access, implementation of ultrasound-guided peripheral intravenous (USGPIV) access in the ED was initiated. Multiple staff from all shifts were trained in the use of the ultrasound.

Dychter, Gold, Carson, and Haller (2012) suggested, $85 \%$ of hospitalized patients receive IV therapy and $75 \%$ of nursing time is spent providing those services. The study recognized problems with PIV access including the 
geriatric population, obesity, dark skin, drug abusers, venous depletion and limited extremity availability. The authors noted, "complications of PIV therapy are costly in terms of patient quality of life, morbidity, morality and treatment expense" (p. 90).

The development of vascular assess decisions in the emergency room (VADER) protocol helps emergency department staff predict the probability of a successful first attempt PIV. This VADER score could direct the most experienced nurse to attempt a difficult PIV to prevent access fail. This study also considered procedural success as prevention of post insertion PIV failure (Carr et al., 2016b).

Carr et al. (2016a) study revealed three intentions, which are to "determine first-time insertion success incidence for PIV in adults, clinician rational for PIV use, and to understand the comparative predictive value of patient and clinician factors that contribute to first-time insertion success" (p. 183). They concluded that achieving greater first-time PIV success would save money and positively impact patient satisfaction. Carr et al. identified that clinicians with the greatest procedural expertise increased the likelihood of PIV success (p. 188). They also noted that infection prevention strategies were needed to reduce the number of insertions and attempts and reduce the idle or unused PIV. Univariate statistics provided the following variables: "patient size $(40 \%$ emaciated, $85 \%$ underweight, $91 \%$ normal, $88 \%$ overweight and $78 \%$ obese); visible veins included (91\% yes, $73 \%$ no), palpable vein ( $88 \%$ yes, $60 \%$ no); cannulation site ( $80 \%$ forearm, $89 \%$ hand, and $89 \%$ cubital fossa); and diameter of vein $(76 \%, 0-2 \mathrm{~mm}, 89 \%>2-3 \mathrm{~mm}, 88 \%>3-4 \mathrm{~mm}$, and $95 \%>4 \mathrm{~mm}$ ) were identified" (Carr et al., 2016a, p. 185).

Moore (2013) demonstrated program development guidelines for USGPIV in an ED and a competency check-off list for registered nurses (RN) in USGPIV insertion. RN's were considered trained and able to perform USGPIV after successful completion of the course. This program noted budgetary considerations. The costs included the participation of all full-time employee's (FTE) with classroom and a 4-hour hands-on practicum. A purchase of an ultrasound gel-training block, which is $\$ 500$. A quality ultrasound machine costs approximately $\$ 30,000$ (Moore, 2013).

Salleras-Duran, Fuentes-Pumarola, Bosch-Borras, Punset-Font, and Sampol-Granes (2016) noted that the USGPIV was successful in $95.1 \%$ of patients $(n=98)$. Successful cannulations were reported at $84.2 \%$ with one attempt. "The mean number of attempts needed to place a catheter was 1.18 (SD, 0.43 ; range, 1 to 3 ) for this study" (p. 340). These results are similar to others (Maiocco \& Coole, 2012; Oliveira \& Lawrence, 2016).

Adhikari et al. (2015) concluded that focused simulation training session for ED nurses supported the use of USGPIV techniques. The study suggested utilization of USGPIV for all patients with known or suspected difficult IV access. Also, USGPIV should be a mandatory skill for all ED nurses (Adhikari et al., 2015).

In a position statement from the Emergency Nurses Association (ENA) 2011 development committee, utilization of USGPIV as a viable option for patients with known difficult access for both adult and pediatric populations received a high recommendation. The usefulness of USGPIV by either an ED physician, ED nurse or an ED technician was endorsed by the ENA with a high recommendation. However, the ENA did not support USGPIV as a more successful route for external jugular access when it is not visible (Crowley, 2012).

\subsection{Theoretical Review}

The literature does not clearly demonstrate a current theoretical model to base this review. The theme that is most prevalent is nursing competence and confidence with the USGPIV technique. This could be incorporated as following Benner's Novice to Expert model. The field of nursing research and utilization created action models. These systematic approaches had been developed to prevent incomplete implementation of findings. Nilsen (2015) espoused interest in the use of theories, models, and frameworks to gain insight into the mechanisms by which implementation is more likely to succeed, has generated a new science. The identification of those at most risk from PIV multiple attempts and need for immediate access lends to using USGPIV as a best practice model. Upon a new CINAHL search with keywords nursing action process, knowledge translation, a model was discovered entitled the Model of Practical Skill Performance ([MPSP] Bjørk, 2013).

\subsection{Summary of Literature Review}

An emergency nurse training program could reduce multiple attempts at PIV access and promote the utilization of USGPIV. The USGPIV training course could be designed to facilitate difficult PIV access patients in the ED. USGPIV access is a popular and highly recommend approach with $87 \%-95 \%$ success rates for difficult PIV patients (Witting, 2012). This type of program could improve patient throughput and improve patient care, treatment and satisfaction.

In review, patients need PIV access in the emergency department for laboratory draw, medications, and fluid 
resuscitation. Difficult to access patients receive multiple PIV attempts by multiple nurses. Patient care is delayed. The use of a predictive scale may help identify patients at risk for difficult PIV access. Emergency nurses trained in the use of USGPIV can be a successful procedure to ensure patient satisfaction, timely patient care, and nursing productivity.

\section{Conclusion}

A gap exists in the use of USGPIV in the ED. Therefore, the application of an A-DIVA score to implement USGPIV access may be beneficial. The A-DIVA prediction tool may improve the success rate of peripheral vascular access in adults rated to have difficult vascular access. The ultrasound-guided technique can be demonstrated using the A-DIVA scoring tool as the determinative factor. Research studies on this important topic are recommended.

\section{References}

Adhikari, S., Schmier, C., \& Marx, J. (2015). Focused simulation training: emergency department nurses' confidence and comfort level in performing ultrasound-guided vascular access. Journal of Vascular Access, 16(6), 515-520. https://doi.org/10.5301/jva.5000436

Bjørk, I.T., Lomborg, K., Nielsen, C.M., Brynildsen, G., Frederiksen, A.M.S., Larsen, ... Stenholt, B. (2013). From theoretical model to practical use: An example of knowledge translation. Journal of Advanced Nursing, 69(10), 2336-2347. https://doi.org/10.1111/jan.12091

Carr, P.J., Rippey, J.C.R., Budgeon, C.A., Cooke, M.L., Higgins, N., \& Rickard, C.M. (2016a). Insertion of peripheral intravenous cannulae in the emergency department: Factors associated with first time insertion success. Journal of Vascular Assess, 17(2), 182-190. https://doi.org/10.5301/jva.5000487

Carr, P.J., Rippey, J.C.R., Cooke, M.L., Bharat, C., Murray, K., Higgins, N.S., \& Rickard, C.M. (2016b). Development of a clinical prediction rule to improve peripheral intravenous cannulae first attempt success in the emergency department and reduce postinsertion failure rates: The Vascular Access Decisions in the $\begin{array}{lllllll}\text { Emergency Room (VADER) study protocol. BMJ Open, } & 6, & \text { 1-7. }\end{array}$ https://doi.org/10.1136/bmjopen-2015-009196

Crowley, M., Brim, C., Proehl, J., Barnason, S., Leviner, S., Lindauer, C., ... Storer, A. (2012). Emergency nursing resource: Difficult intravenous access. Journal of Emergency Nursing, 38(4), 335-343. https://doi.org/10.1016/j.jen.2012.05.010

Dychter, S.S., Gold, A.A., Carson, D., \& Haller, M. (2012). Intravenous therapy: A review of complications and economic considerations of peripheral access. Journal of Infusion Nursing, 35(2), 84-91. https://doi.org/10.1097/NAN.0b013e31824237ce

Maiocco, G., \& Coole, C. (2012). Use of ultrasound guidance for peripheral intravenous placement in difficult-to-access patients. Journal of Nursing Care Quality, 27(1), 51-55. https://doi.org/10.1097/NCQ.0b013e31822b4537

Moore, C. (2013). An emergency department nurse-driven ultrasound-guided peripheral intravenous line program. Journal of the Association for Vascular Access, 18(1), 45-51. https://doi.org/10.1016/j.java.2012.12.001

Nilsen, P. (2015). Making sense of implementation theories, models, and frameworks. Biomed Central, 1-13. https://doi.org/10.1186/s13012-015-0242-0

Oliveira, L., \& Lawrence, M. (2016). Ultrasound-guided peripheral intravenous access program for emergency physicians, nurses, and corpsmen (technicians) at a military hospital. Military Medicine, 181, 272-276. https://doi.org/10.7205/MILMED-D-15-00056

Pandurangadu, A.V., Tucker, J., Bagan, M., \& Bahl, A. (2016). Patient satisfaction with nurse placed ultrasound guided peripheral IV. Emergency Medicine, 6(3), 1-4. https://doi.org/10.4172/2165-7548.1000325

Petroski, A., Frisch, A., Joseph, N., \& Carlson, J.N. (2015). Predictors of difficult pediatric intravenous access in the community emergency department. Journal of Vascular Access, 16(6), 521-526. https://doi.org/10.5301/jva.5000411

Salleras-Duran, L., Fuentes-Pumarola, C., Bosch-Borras, N., Punset-Font, X., \& Sampol-Granes, F.X. (2016). Ultrasound-guided peripheral venous catheterization the emergency services. Journal of Emergency Nursing, 42(4), 338-343. https://doi.org/10.1016/j.jen.2015.11.005

Sou, V., McManus, C., Mifflin, N., Frost, S.A., Ale, J., \& Alexandrou, E. (2017, November). A clinical pathway 
for the management of difficult venous access. BioMed Central, 1-7. https://doi.org/10.1186/s12912-017-0261-z

Van Loon, F.H.J., Puijn, L.A.P.M., Houterman, S., \& Bouwman, A.R.A. (2016). Development of the A-DIVA scale: A clinical predictive scale to identify difficult intravenous access in the adult patients based on clinical observation. Medicine, 95(16), 1-8. https://doi.org/10.1097/MD.0000000000003428

Witting, M.D. (2012). IV access difficulty: Incidence and delays in an urban emergency department. Journal of Emergency Medicine, 42(4), 483-487. https://doi.org/10.1016/j.jemermed.2011.07.030

\section{Copyrights}

Copyright for this article is retained by the author(s), with first publication rights granted to the journal.

This is an open-access article distributed under the terms and conditions of the Creative Commons Attribution license (http://creativecommons.org/licenses/by/4.0/). 